\title{
Gamma-ray-based measurement of concentration distribution in pipe flow of settling slurry: vertical profiles and tomographic maps
}

\author{
Jan Krupička*, Václav Matoušek \\ Institute of Hydrodynamics of the Academy of Sciences of the Czech Republic, v. v. i., Pod Pat'ankou 30/5, 166 12, Prague, Czech Repub- \\ lic. \\ ${ }^{*}$ Corresponding author. E-mail: krupicka@ih.cas.cz
}

\begin{abstract}
Principles of gamma-ray-based measurement are summarized and their application is demonstrated on an operation of the radiometric facility installed in the test loop for slurry flows at the Institute of Hydrodynamics. The facility is able to measure vertical profiles of chord-averaged concentrations and concentration maps in the pipe cross section. A methodology of measurement is proposed including detection and quantification of random and systematic errors. Experimental results are discussed in the light of the proposed methodology. Experimentally determined vertical profiles of concentration are presented for slurry flows of four different fractions of glass beads. The tomographic application of the radiometric device is demonstrated on a measured concentration map and a suitable image reconstruction method is tested. High reliability of measured concentration distributions is proved except for regions near the pipe wall. The radiometric method is shown to be a useful tool for measurement of concentration distribution in slurry flow through a pipe.
\end{abstract}

Keywords: Two-phase flow; Gamma radiometry; Computational tomography; Slurry flow experiment.

\section{INTRODUCTION}

Various methods and techniques have been developed and tested for a measurement of local concentration of solids in solid-liquid flows through pipes. Examples are isokinetic sampling (Nasr-el-din et al., 1984), electrical-resistance-based methods (Graham et al., 2002; Sutherland et al., 2008), and radiometry-based methods (e.g. Gillies, 1993; Shook and Daniel, 1965). A principle of the gamma-ray-based method is similar to the principle of well-known RTG - the intensity of radiation passing through the investigated matter, $I$, depends on the initial intensity of radiation, $I_{0}$, and on the average value of the attenuation coefficient along the beam of the length $L$, $1 / L \cdot \int_{L} \mu \cdot d l$, where $\mu$ is the local attenuation coefficient for gamma ray in penetrated matter. The exponential relationship reads

$$
I / I_{0}=e^{-\int \mu \cdot d l} .
$$

The attenuation coefficient is affected by the atomic structure of penetrated matter and a value of the coefficient is related to the density of the matter. This condition predestines the method for use in mixtures composed of phases of significantly different densities.

Specific configurations of gamma-ray-based systems can be different. In slurry pipes, a measuring system composed of one source of a gamma-ray beam on one side and one detector on the opposite side of a pipe cross section was successfully applied to obtain a vertical profile of chord averaged concentrations in the pipe cross section by vertical shifting of a sourcedetector position (e.g. Gillies, 1993; Matoušek, 1997; Pugh, 1995). More complex configurations with more than one gamma-ray source and/or a number of detectors are often employed in gamma-ray tomography systems for research and industrial applications. Such devices enable to screen objects from different positions and angles, and to reconstruct maps of density or concentration in the measuring volume over a very short period of time (e.g. Hjertaker and Johansen, 2008). The number of sources and detectors can be reduced if those are allowed to rotate round the measuring volume (e.g. Hampel et al., 2007; Lee et al., 2009). One source and one detector only can be also employed to provide tomographic data provided that their linear and radial shifting is allowed and combined. The disadvantage is that much longer measuring time is required to produce a map. Przewlocki et al. (1979) used such a system to reconstruct concentration maps in a cross section of a slurry pipe and the same configuration was selected for radiometric devices installed to our experimental pipe loop at the Institute of Hydrodynamics in Prague.

\section{EXPERIMENTAL METHODOLOGY Experimental device and procedure}

Since 2012, a completely re-modelled test loop for slurry flow investigations has been in full operation at the Institute of Hydrodynamics in Prague. Loop pipes are made of stainless steel and have an inner diameter $D=100 \mathrm{~mm}$. Detailed information about the loop and its measurement facilities is available in Matoušek et al. (2014). Two radiometric devices are parts of the test loop. Fig. 1 shows a schematic view of the radiometric devices. Radioactive isotope of caesium, Cs-137, serves as a gamma-ray source at one side of the pipe. When the aperture is opened in the source shielding, radiation passes through the adjustable opening in a lead collimator and penetrates the pipe in the form of a diverging beam. During our experiments presented below, the opening was adjusted to the height of 1.5 $\mathrm{mm}$. The spatial resolution of a measurement is further increased by another lead collimator installed in the detector at the opposite side of the pipe. The 1-mm narrow fixed opening allows that only the central portion of the beam reaches the detector and eliminates photons reflected from other directions than from the source. An analyser sorts electrical impulses on the basis of the energy of incident gamma photon. Only events with the energy close to that of Cs-137 primary emission (662 $\mathrm{keV}$ ) are counted and others (caused by reflected and spread photons from the source and by natural radioactive background) are filtered out. The output of the measurement is the count of 
photons that passed from the source to the detector ideally without any interaction with interstitial matter. Depending on source collimator opening, values of the initial intensity $C P S_{0}=$ $3000-5000 \mathrm{~s}^{-1}$ (CPS = Counts Per Second) are typical for cases of no attenuation (i.e. no penetrated matter) between the source and the detector. A position of both the source and the detector is set by the PC-controlled linear positioning drive. A user predefines the table of vertical positions above or below pipe centre, the computer goes through the table, sets the device to the positions, and carries out measurements at the positions. The procedure produces a set of parallel-projection data from which a profile of chord-average concentrations is constructed. The linear positioning drive is combined with the manually handled radial positioning drive. This makes it possible to install and vary inclination angles at which parallel projections are sensed.



Frontal view of collimator


Fig. 1. Scheme of radiometric device at Institute of Hydrodynamics in Prague. 1 - gamma-ray source; $2 \mathrm{a}$ - collimator at source; $2 \mathrm{~b}-$ collimator at detector; 3 - scintillator; 4 - photomultiplier; 5 digital analyser; 6 - lead shielding of detector; 7 - pipe cross section; 8 - linear positioning drive; 9 - radial positioning drive (Krupička and Matoušek, 2012).

\section{Evaluation of chord-averaged concentrations}

Data collected from parallel projections have a form of a set of vertical positions, $y$, and corresponding counts of photons, $N$, detected at $y$ over the measuring time period, $T$. As the first step, $N$ is divided by $T$ to get the intensity $C P S$. Measured attenuation of radiation at $y$ is expressed as the ratio $C P S_{n}=$ $C P S / C P S_{0}$, where $C P S_{0}$ has to be determined from a calibration measurement carried out outside the pipe. If $C P S_{n}$ is assigned to the left side of Eq. (1), the mean attenuation coefficient can be calculated from the right side of the equation. However, Eq. (1) is exactly valid only in the ideal case of infinitely narrow beam attenuated in obstacle between infinitely distanced source and detector while the resulting value of the coefficient involves also attenuation due to pipe wall and an effect of complex geometry. To minimize these effects, a calibration needs to be carried out for two limiting cases: 1 . in pipe filled with fluid only and 2 . in pipe filled with mixture at maximum concentration of solids (i.e. at the concentration of loose poured material $c_{b}$ ). In each case, a profile of $C P S_{n, f}, C P S_{n, b}$ respectively, is sensed in the pipe cross section (Fig. 2a). If the densities of both fillings are known ( $\rho_{f}$ for fluid and $\rho_{b}$ for mixture of concentration $c_{\mathrm{b}}$ ), then the actual chord-averaged density $\bar{\rho}_{m}$ can be calculated using a logarithmic interpolation at each position $y$ (Fig. 2b). Finally, a chord-averaged concentration is calculated from the evaluated density as $\bar{c}=\left(\bar{\rho}_{m}-\rho_{f}\right) /\left(\rho_{s}-\rho_{f}\right)$, where $\rho_{s}$ is the density of solids (Fig. 2c, d).

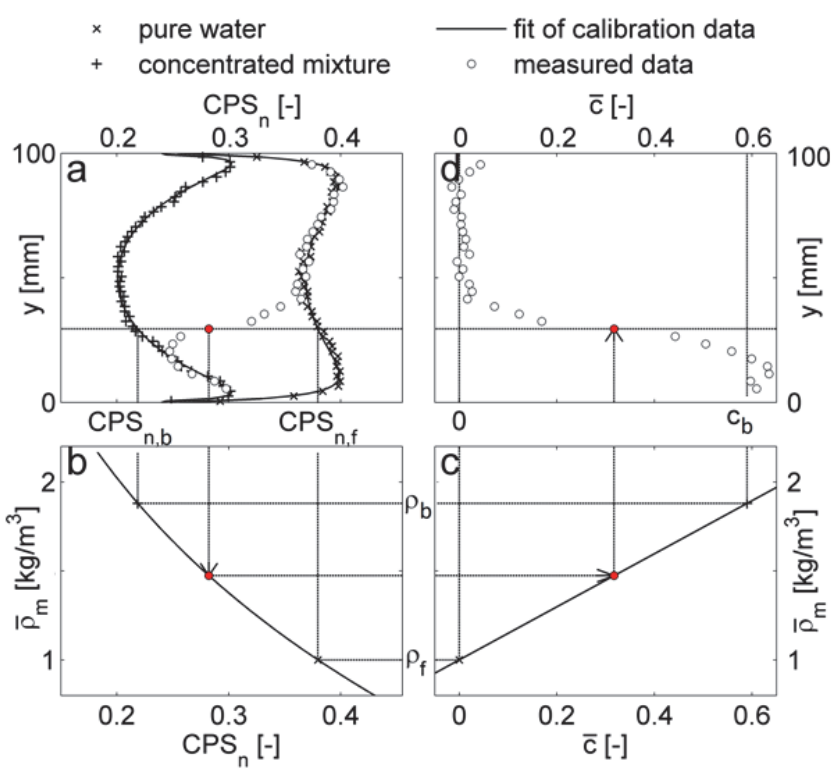

Fig. 2. Process of radiometric data evaluation - a) profiles of normalised CPS for measured data and for calibration made with water and concentrated mixture; b) logarithmic interpolation of density at selected position $y$; c) linear interpolation of solids concentration; d) evaluated profile of chord-averaged concentration.

\section{Evaluation of local concentrations}

Parallel projections can be collected at several angles of inclination and the procedure described above applies to all parallel projections. Acquired density profiles can be processed further by methods of computer tomography (CT) to determine local densities within the pipe cross section. Various CT methods can be found in the literature (see e.g. Kak and Slaney, 1988). An appropriate image reconstruction method must be selected with regard to the amount of data that need to be processed to the computational effort in relation with desired accuracy and to the nature of imaged phenomena.

Measurement of one projection using our radiometric devices is quite time consuming, measurement of the entire set of projections can take tens of minutes. Therefore, the total amount of data we collect during a test run is limited. The measuring time affects also a choice of an image reconstruction method. Since the measuring time is much longer than the computational time of any reconstruction method, we can afford a method that is usually considered time consuming - the SIRT (Simultaneous Iterative Reconstruction Technique, Gilbert, 1972) method which belongs to a group of algebraic image reconstruction methods. An advantage of this choice is that, contrary to the methods based on Fourier transformation, the algebraic methods do not require a constant step between projections (Kak and Slaney, 1988). A majority of reconstructive techniques uses a pixel-based representation of a density distribution with the constant value of density within each pixel. This representation is inappropriate in our application for two reasons: our pipe is of a circular perimeter and gradual changes in local density are expected rather than sharp interfaces in pipe flow of slurry. Rectangular pixels can accommodate to these conditions properly only if very fine elements are used and hence many unknowns are introduced. A use of a triangular mesh and a linear interpolation of density from values stored in 
nodes (Fig. 3) reduce a number of unknowns. We also tested a polynomial representation (Krupička and Matoušek, 2012) as used by Przewlocki et al. (1979) and a representation by Fourier series and did not find any benefit in using those approaches instead of the one with the triangular mesh.

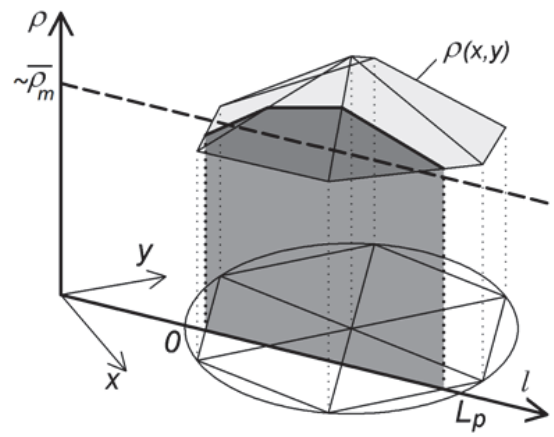

Fig. 3. Piecewise linear approximation of density distribution defined by densities stored in nodes of triangular mesh. Mean density along beam axis is given as a product of an intersection of beam with the approximation.

Fig. 3 indicates how the system of algebraic equations is established for unknown densities stored in nodes of the triangular mesh. For each individual measurement, an approximation of density distribution $\rho(x, y)$ is integrated over the beam length inside the pipe, $L_{P}$. As a result of the integration, approximation of mean density is expressed by a linear combination of unknown densities and it is set equal to the mean density evaluated from the measurement, $\bar{\rho}_{m}$. A sufficiently detailed density map requires the number of nodes higher than the number of individual measurements obtained over the reasonably long measuring time. Thus, one obtains an undetermined system of linear algebraic equations which is solved iteratively by the SIRT algorithm.

\section{Estimation of measuring errors}

Possible sources of measuring errors were discussed by Krupička and Matoušek (2012). The natural fluctuation in gamma-photon emission and detection was identified as a major contributor to the measuring error. In principle, detected intensity of gamma-ray radiation is a stochastic variable with a Poisson distribution. A distribution of random deviations from the mean value of intensity can be approximated by the normal distribution with the standard deviation $\sigma_{C P S}=\sqrt{C P S / T}$. For a given radiometric device (i.e. $C P S_{0}=$ const.), these deviations can be damped only by increasing the measuring time period $T$. Other errors arise from inaccuracy of the linear and radial positioning and from simplifications associated with data evaluation; the assumption of an infinitely narrow beam and the assumption of exact validity of Eq. (1). Nevertheless, our simulations of a measuring process showed that these errors were of minor importance except for the regions close to the pipe wall (Krupička and Matoušek, 2012). This result agrees with our experimental experience.

The same procedure as in Krupička and Matoušek (2012) is applied to quantify accuracy of profiles of chord-averaged concentration. For a hypothetical profile (Fig. 4a), a number of simulated measurements and evaluations of data is performed. All potentially error-causing effects mentioned in the previous paragraph are introduced to the simulations and values corre- sponding with our experimental experience are used - the height of beam is set to the value of $1 \mathrm{~mm}$, errors in linear positioning drive are generated randomly assuming the normal distribution with the standard deviation of $0.32 \mathrm{~mm}, C P S_{0}=$ $4700 \mathrm{~s}^{-1}$ and $T=8 \mathrm{~s}$. As a result, a concentration profile different from that in Fig. $4 \mathrm{a}$ is obtained from each simulation. Differences from 5000 simulations are processed to calculate a profile of standard deviations plotted in Fig. 4b. Fig. $4 \mathrm{~b}$ gives a picture of expected random errors in measurements of chordaveraged concentration profiles at the given experimental conditions.

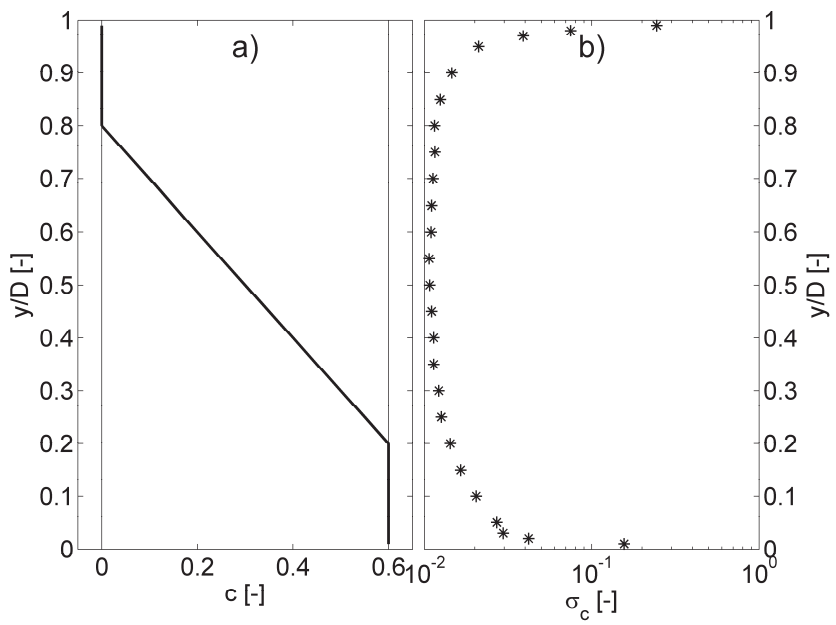

Fig. 4. a) hypothetical concentration profile used for numerical simulations of measurement and data evaluation; b) profile of standard deviations of concentration calculated from simulated data corrupted by random errors.

Besides the random errors discussed above, an attention must be paid also to the systematic error arising from fluctuations of concentration in flowing slurry during the measuring time $T$. It is well known (e.g. Hampel and Wagner, 2011) that fluctuations result in an underestimation of a measured value of the chord-averaged concentration. To estimate a magnitude of this type of an error, let us assume the chord-averaged concentration to be composed of the time-averaged and the fluctuating components $\bar{c}(t)=\bar{c}+\bar{c}^{\prime}(t)$. The derived relationship for the systematic error in measured concentration reads

$e_{c}=\frac{c_{b}}{L_{P} \cdot\left(\mu_{b}-\mu_{f}\right)} \cdot \ln \left[1 / T \cdot \int_{0}^{T} \mathrm{e}^{-L_{P} \cdot\left(\mu_{b}-\mu_{f}\right) \cdot \cdot^{\prime}(t) / c_{b}} d t\right]$,

where $\mu_{\mathrm{b}}$ and $\mu_{\mathrm{f}}$ are the attenuation coefficients for mixture of concentration $c_{\mathrm{b}}$, fluid respectively. The specific form of the function $\bar{c}^{\prime}(t)$ is unknown and it has to be assumed. Switching between two limits of $\pm \Delta c$ is the simplest model for $\bar{c}^{\prime}(t)$ and the most unfavourable at the same time, so it can be used to calculate the upper limit of possible errors. Eq. (2) fed by $\bar{c}^{\prime}(t)= \pm \Delta c$ and experimentally determined values of $\left(\mu_{b}-\mu_{f}\right) \approx 6.5 m^{-1}$ and $c_{b} \approx 0.6$ produces the profile of errors plotted in Fig. 5. The maximum value of $\Delta c$ is $c_{b} / 2 \approx 0.3$ because $\bar{c}(t)$ must lie within the range $\left(0, c_{b}\right)$. At this limit, the maximum value of the calculated error in concentration $e_{\mathrm{c}}=$ 0.05 in the pipe centre and the error decreases towards the pipe wall reaching zero at the wall (Fig. 5). An assumption of a 
sinusoidal course of $\bar{c}^{\prime}(t)$ is probably more reasonable and our calculations showed that it produces less than half of the errors presented in Fig. 5. Usually, a value of $\Delta c$ is much smaller than $c_{b} / 2$ so that the errors due to fluctuations $e_{\mathrm{c}}$ are smaller than 0.01 and can be neglected.



Fig. 5. Profile of systematic errors due to fluctuation of chordaveraged concentration. Lines represent errors calculated for different values of fluctuation amplitude $\Delta c$.

\section{DISCUSSION OF EXPERIMENTAL RESULTS}

Four fractions of narrow graded artificial glass beads (ballotini) were tested in mixture with water. Table 1 summarizes experimentally determined values of particle mean diameter, $d_{50}$, density, $\rho_{s}$, loose poured concentration, $c_{b}$, and settling velocity of mean particle, $w_{t, 50}$. For each fraction, experiments were carried out at three different average concentrations given by the total amount of solids in the pipe loop. Test runs started at the average velocity $V_{m}$ slightly above the deposition limit and then $V_{m}$ was gradually increased, usually up to $4 \mathrm{~m} / \mathrm{s}$.

Table 1. Properties of tested solids.

\begin{tabular}{lcccc}
\hline acronym & $\begin{array}{c}d_{50} \\
10^{-3}[\mathrm{~m}]\end{array}$ & $\begin{array}{c}\rho_{\mathrm{s}} \\
10^{-3}\left[\mathrm{~kg} \cdot \mathrm{m}^{-3}\right]\end{array}$ & $\begin{array}{c}c_{\mathrm{b}} \\
{[-]}\end{array}$ & $\begin{array}{c}w_{\mathrm{t}, 50} \\
10^{-2}[\mathrm{~m} / \mathrm{s}]\end{array}$ \\
\hline B9 & 0.44 & 2.46 & 0.61 & 5.4 \\
B8 & 0.53 & 2.46 & 0.61 & 7.1 \\
TK0810 & 0.90 & 2.57 & 0.58 & 14.6 \\
TK1516 & 1.55 & 2.49 & 0.61 & 22.3 \\
\hline
\end{tabular}

\section{Vertical concentration profiles}

Measured vertical profiles of chord-averaged concentrations are plotted for different tested fractions of solids in Figs. 6-9. For measurements of the profiles, the installed vertical step was $2 \mathrm{~mm}$. A simple central moving average over four points was applied on the concentration data to produce smoothed profiles (dashed lines in Figs. 6-9) and to make an evaluation of a scatter of the data possible.
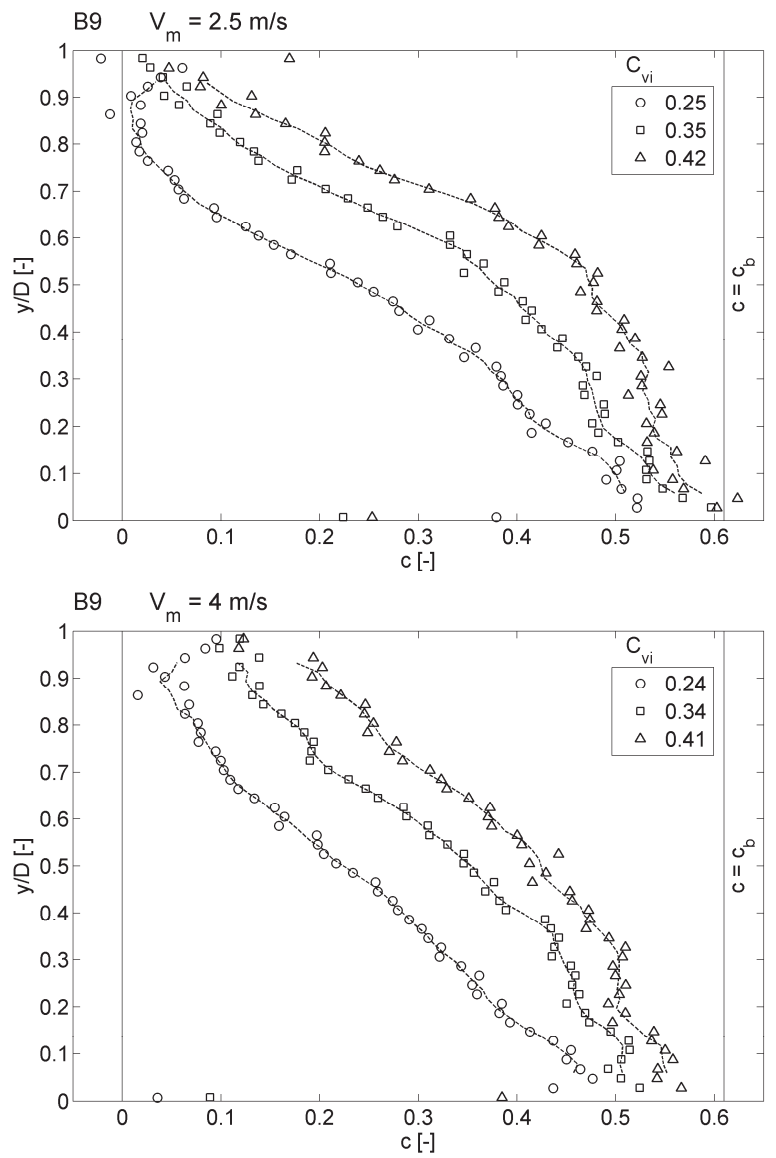

Fig. 6. Vertical profiles of chord-averaged concentrations in mixture of water and B9 at different mean velocities $V_{m}$ and concentrations $C_{v i}$. 

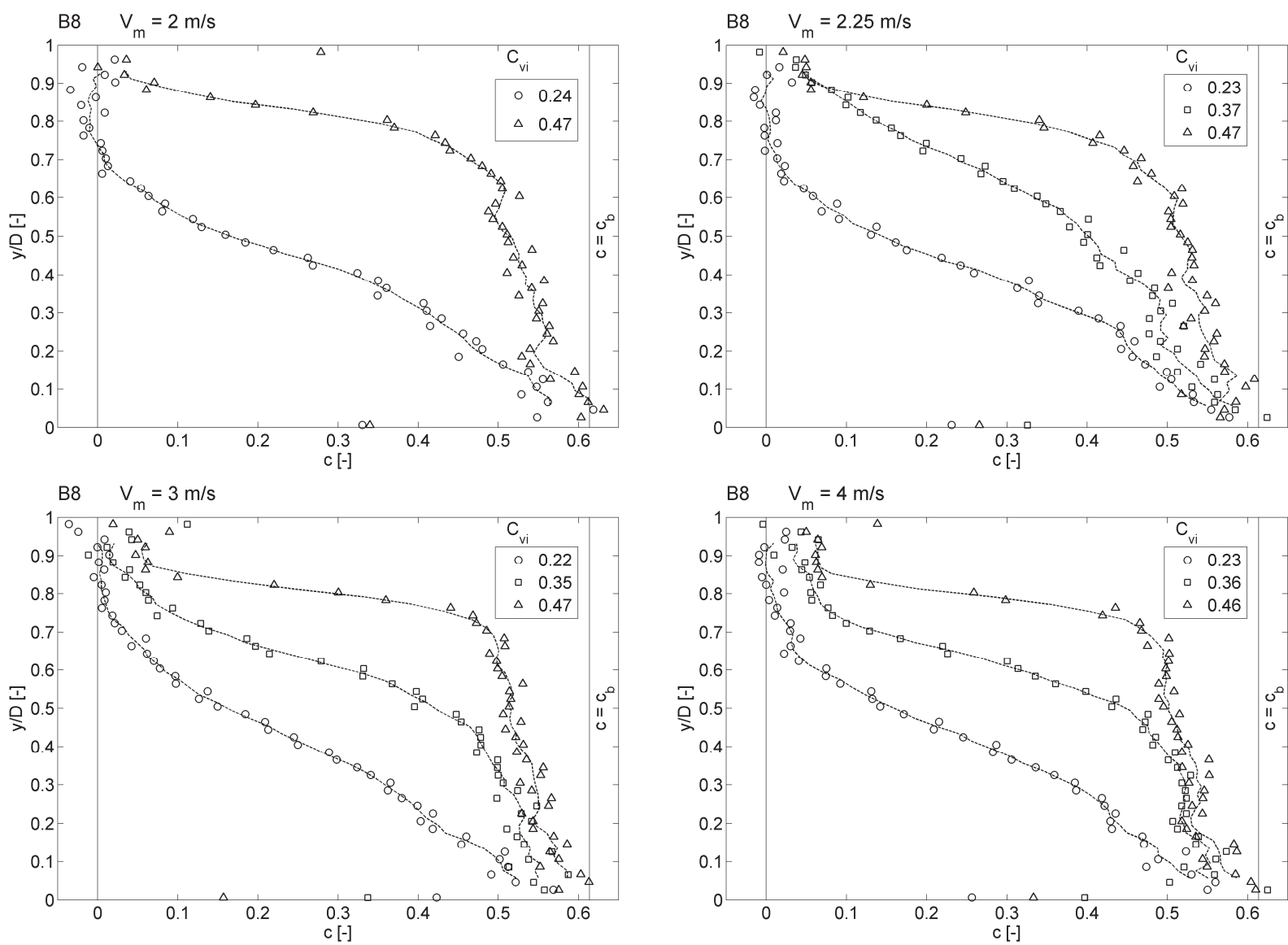

Fig. 7. Vertical profiles of chord-averaged concentrations in mixture of water and $\mathrm{B} 8$ at different mean velocities $V_{m}$ and concentrations $C_{v i}$.
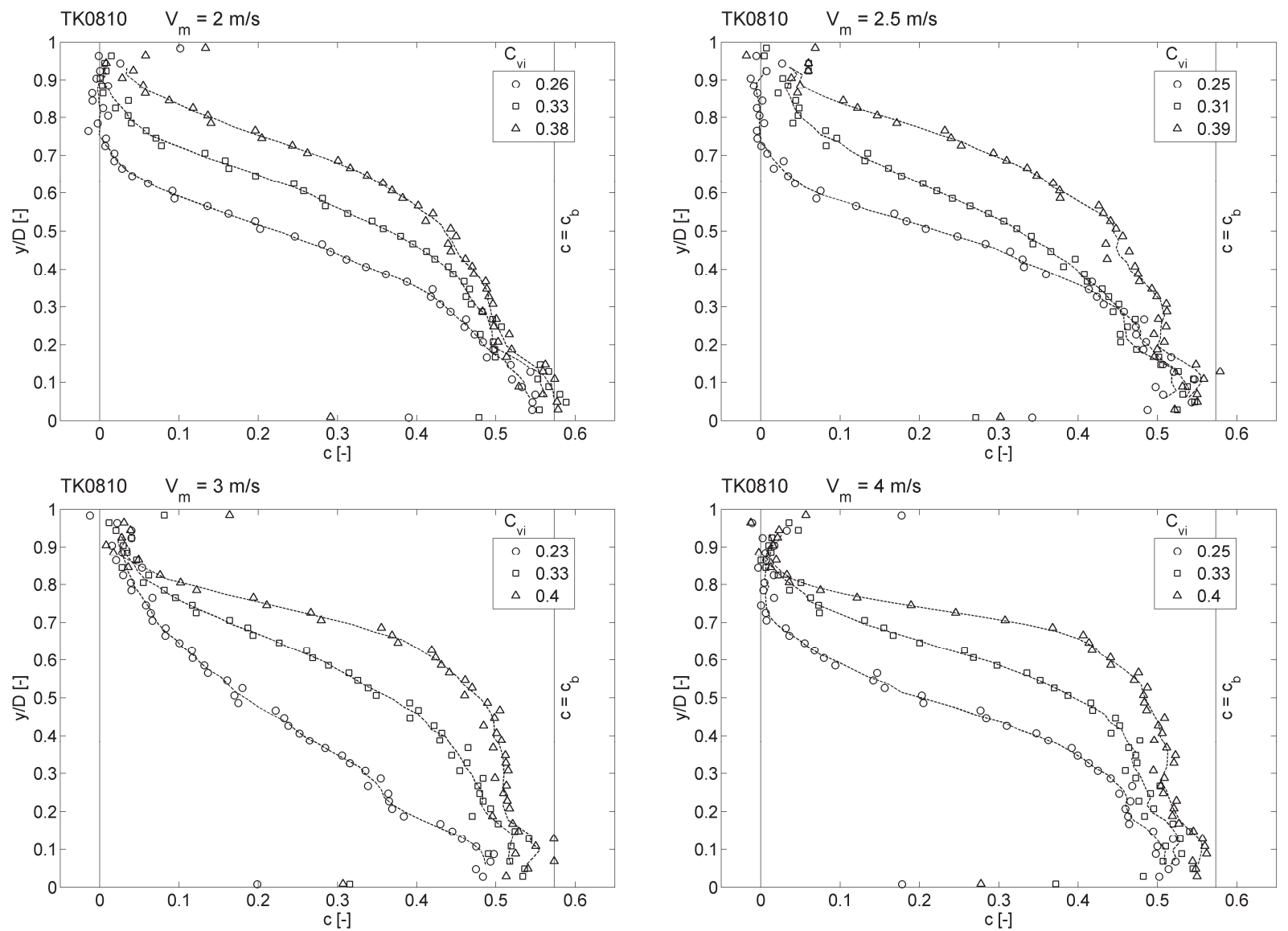

Fig. 8. Vertical profiles of chord-averaged concentrations in mixture of water and TK0810 at different mean velocities $V_{m}$ and concentrations $C_{v i}$. 

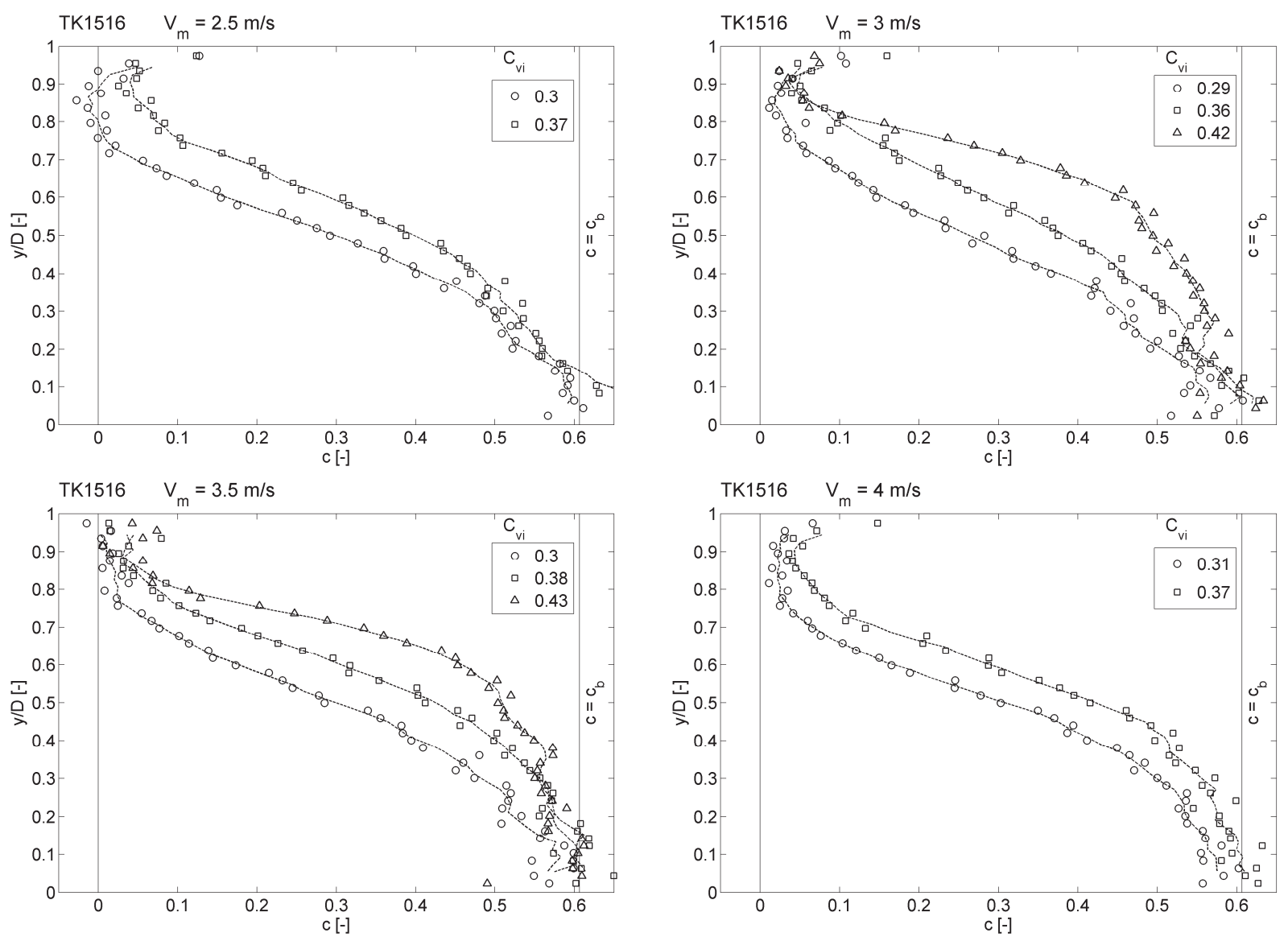

Fig. 9. Vertical profiles of chord-averaged concentrations in mixture of water and TK1516 at different mean velocities $V_{m}$ and concentrations $C_{v i}$.

The measuring time period was installed to 8 seconds at each position. For a majority of the measurements, this was long enough to average out most of local flow fluctuations with as a result a small scatter of the data in concentration profiles (except of the regions near the pipe wall where the measuring errors of the radiometric method rise considerably as discussed earlier). A very similar scatter was experienced in low and high-concentrated regions in the flow and also the size of particles did not seem to affect the scatter significantly (compare different profiles at $C_{v i}=0.22-0.26$ in Figs. 6-8). These observations agree well with the theoretical analysis of measuring errors. There were some profiles, however, in which the observed scatter was too big to be explained by measuring errors (lower portions of most of profiles at $C_{v i}>0.4$ in Figs. 6-9). In those cases, perhaps the time scale of local flow fluctuations was comparable to the measuring time period.

Measurement of a concentration profile in water (i.e. $c=0$ ) was performed to quantify measuring errors experimentally. The obtained concentrations were scattered around the mean value of -0.01 with the standard deviation of 0.01 within the inner region of pipe $(y / D=0.2-0.8)$. This systematic error cannot be attributed to fluctuations and its origin remains unclear at the moment. Nevertheless, $e_{\mathrm{c}}=0.01$ is acceptable and the standard deviation agrees well with numerical results presented in Fig. 4.

\section{Map of local concentrations}

Due to long measuring time required by tomographic data acquisition, this application was not used regularly, only vertical profiles were sensed during experiments presented above.
The tomographic application of the radiometric device was tested only. Fig. 10 shows results of the SIRT algorithm applied on the data published earlier (Krupička and Matoušek 2012) with the mixture of water and TK0810 flowing above an eroded surface of stationary bed in a $100-\mathrm{mm}$ pipe. The tomographic data used in Fig. 10 consist of 18 parallel projections at inclinations of $0^{\circ}-170^{\circ}$ (in a step of $10^{\circ}$ ) and parallel beams are in a step of $10 \mathrm{~mm}$. As a result, 202 of individual measurements were processed. Count of pulses collected at the each position was of the order of magnitude of $10^{4}$. The triangular mesh consist of 331 nodes and cells of the characteristic size of $D / 20$ what seems to be enough to approximate the density map in sufficient details. 25 iterations were performed to produce the presented tomogram. It can be seen that the evaluated position of the top of the bed agrees well with the visual observation. The transport layer with a gradually decreasing concentration above the bed is also identified although higher uniformity in the horizontal direction would be expected and the observed undulation is probably an artefact caused by the measurement and the image reconstruction. This is also the case for local non-zero concentrations (low positive values) in the upper part of the pipe cross section. Considerable errors are produced in the vicinity of pipe wall and near the bed surface in particular. These were reproduced by all of the reconstruction methods tested up to date and have to be considered the greatest weakness of the measuring technique. 


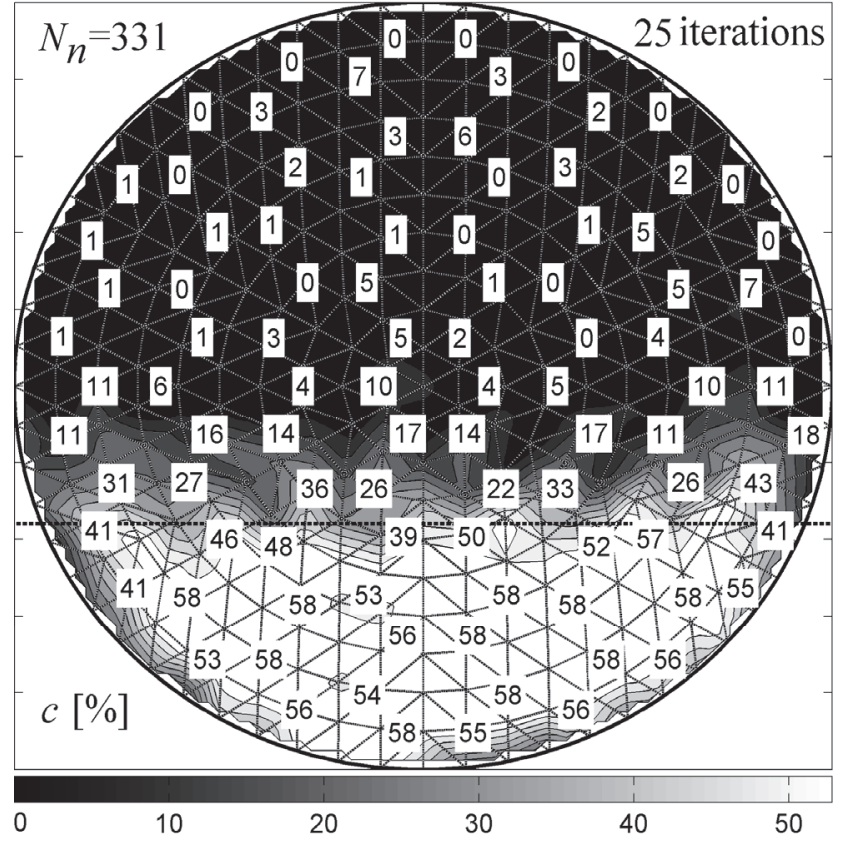

Fig. 10. Distribution of local volumetric concentration (in per cent) of TK0810 in mixture with water flowing above a stationary bed $\left(V_{\mathrm{m}}=1.0 \mathrm{~m} / \mathrm{s}, C_{\mathrm{vi}}=0.21\right)$. Dashed line - position of bed observed visually in a transparent section of the pipe.

\section{CONCLUSIONS}

The radiometric device installed in the 100-mm-pipe loop at the Institute of Hydrodynamics in Prague was employed in experimental investigations on an inner structure of settling slurry flows. Vertical profiles of chord-averaged concentrations were measured in slurry flows of four different fractions of glass beads (particles sizes from $0.4 \mathrm{~mm}$ to $1.6 \mathrm{~mm}$ ). An analysis of measuring errors is a part of the presented methodology for radiometric measurements of concentration distribution in slurry pipes. The analysis suggests that the random error in measured concentrations does not exceed 0.02 except for the region near the pipe wall. The measurement is not reliable in the vicinity of a pipe wall (say up to $5 \mathrm{~mm}$ from wall). The theoretical estimation of errors agrees well with experimental observations.

Tomographic data sets were collected for the flow above a stationary deposit in the pipe and processed by the SIRT method to evaluate local concentrations within a pipe cross section using a triangular mesh. A total count of 202 individual measurements in 18 sets of parallel projections enabled to reconstruct a concentration map with a sufficient resolution. Nevertheless, considerable errors occurred near the pipe wall. In the inner region of the cross section, the magnitude of local errors could be estimated as 5 to 10 per cent at maximum.

A radiometric method of sensing chord-averaged/local densities is confirmed to be useful and reliable for slurry flows in pipes. The new experimental results contribute to the existing database of concentration profiles in pipe flows of settling slurries.

Acknowledgement. The financial support of the Czech Science Foundation through the grant project No. 103/09/0383 is gratefully acknowledged.

\section{REFERENCES}

Hampel, U., Bieberle, A., Hoppe, D., Kronenberg, J., Schleicher, E., Sühnel, T., Zimmermann, F., Zippe, C., 2007. High resolution gamma ray tomography scanner for flow measurement and non-destructive testing applications. Rev. Sci. Instrum., 78, 10, 103704-1 - 103704-9.

Hampel, U., Wagner, M., 2011. A method for correct averaging in transmission radiometry. Meas. Sci. Technol., 22, 115701.

Hjertaker, B.T., Johansen, G.A., 2008. High speed gamma-ray tomography for hydrocarbon flow applications. An International Conference on the Applications of Computerized Tomography. AIP Conference Proceedings, 1050, 163-174.

Gilbert, P., 1972. Iterative methods for the three-dimensional reconstruction of an object from projections. J. Theor. Biol., $36,1,105-117$.

Gillies, R.G., 1993. Pipeline Flow of Coarse Particle Slurries. $\mathrm{PhD}$ thesis. University of Saskatchewan, Saskatoon, Canada.

Graham, L., Hamilton, R., Rudman, R., Strode, P., Pullum, L., 2002. Coarse solids concentration profiles in laminar pipe flows. Proceedings Hydrotransport 15. Banff, Canada, pp. 149-158.

Kak, A.C., Slaney, M., 1988. Principles of Computerized Tomographic Imaging. IEEE Press.

Krupička, J., Matoušek, V., 2012. Gamma-ray-based method for density sensing in pipes - evaluation of measurement and data processing. Proceedings of the 2nd IAHR Europe Congress, Munich, Germany.

Lee, N.Y., Jung, S.H., Kim, J.B., 2009. Evaluation of the measurement geometries and data processing algorithms for industrial gamma tomography technology. Appl. Radiat. Isot., $67,7-8,1441-1444$.

Matoušek, V., 1997. Flow Mechanism of Sand-Water Mixtures in Pipelines. Doctoral thesis. Delft University Press, Delft, The Netherlands.

Matoušek, V., Krupička, J., Pěník, V., 2014. Distribution of medium-to-coarse glass beads in slurry pipe flow: evaluation of measured concentration profiles. Particul. Sci. Technol., 32, 2, 186-196.

Nasr-el-din, H., Shook, C.A., Esmail, M.N., 1984. Isokinetic probe sampling from slurry pipelines. Can. J. Chem. Eng., 62, 179-185.

Pugh, F.J., 1995. Bed-Load Velocity and Concentration Profiles in High Shear Stress Flows. PhD thesis. Queen's University at Kingston, Kingston, Canada.

Przewlocki, K., Michalik, A., Korbel, K., Wolski, K., Parzonka, W., Sobota, J., Pac-Pomarnacka, A., 1979. A radiometric device for the determination of solids concentration distribution in a pipeline. Proceedings of Hydrotransport 6, Cantenbury, UK.

Shook, C.A., Daniel, S.M. 1965. Flow of suspensions of solids in pipelines: Part I. Flow with a stable stationary deposit. Can. J. Chem. Eng., 43, 2, 56-61.

Sutherland, A.P.N., Long, T.M., Randall, E.W., Wilkinson, A.J., 2008. Determining concentration and velocity profiles of non-Newtonian settling slurries using electrical resistance tomography. J. S. Afr. Inst. Min. Metall., 108, 10, 583-590.

Received 20 December 2013 Accepted 23 January 2014 